\title{
Increased Cytokeratin 19 Fragment Levels Are Positively Correlated with Adenosine Deaminase Activity in Malignant Pleural Effusions from Adenocarcinomas
}

\author{
Jorge Luiz Barillo $\left(\mathbb{D},{ }^{1,2}\right.$ Cyro Teixeira da Silva Junior $\left(\mathbb{D},{ }^{3}\right.$ Patricia Siqueira Silva, ${ }^{4}$ \\ Joeber Bernardo Soares de Souza $\mathbb{D}^{1},{ }^{1,2}$ Salim Kanaan, ${ }^{5}$ Analucia Rampazzo Xavier $\mathbb{D}^{5}{ }^{5}$ \\ and Elizabeth Giestal de Araujo $\mathbb{D}^{6}$ \\ ${ }^{1}$ Antonio Pedro University Hospital, Fluminense Federal University, Niteroi, RJ, Brazil \\ ${ }^{2}$ General Hospital Santa Teresa, Petropolis, RJ, Brazil \\ ${ }^{3}$ Department of Clinics, School of Medicine, Fluminense Federal University, Niteroi, RJ, Brazil \\ ${ }^{4}$ Clinical Analysis Postgraduate Program, Fluminense Federal University, Niteroi, RJ, Brazil \\ ${ }^{5}$ Department of Clinical Pathology, School of Medicine, Fluminense Federal University, Niteroi, RJ, Brazil \\ ${ }^{6}$ Department of Neurobiology, Biology Institute, Fluminense Federal University, Niteroi, RJ, Brazil
}

Correspondence should be addressed to Cyro Teixeira da Silva Junior; ctsilvajunior@predialnet.com.br

Received 15 February 2018; Accepted 29 March 2018; Published 8 May 2018

Academic Editor: Paola Gazzaniga

Copyright (C) 2018 Jorge Luiz Barillo et al. This is an open access article distributed under the Creative Commons Attribution License, which permits unrestricted use, distribution, and reproduction in any medium, provided the original work is properly cited.

\begin{abstract}
Adenosine deaminase (ADA) and cytokeratin 19 (CK19) are known pleural biomarkers. Although ADA in humans functions mainly in the immune system, it also appears to be associated with the differentiation of epithelial cells. Keratin filaments are important structural stabilizers of epithelial cells and potent biomarkers in epithelial differentiation. This study aimed to investigate the simultaneous presence of the ADA enzyme and CK19 fragments to assess epithelial differentiation in malignant and benign pleural fluids. Diagnosis of the cause of pleural effusion syndrome was confirmed by means of standard examinations and appropriate surgical procedures. An ADA assay, in which ADA irreversibly catalyzes the conversion of adenosine into inosine, was performed using a commercial kit. The CK19 assay was performed using a CYFRA 21-1 kit, developed to detect quantitative soluble fragments of CK19 using an electrochemiluminescence immunoassay. One hundred nineteen pleural fluid samples were collected from untreated individuals with pleural effusion syndrome due to several causes. ADA levels only correlated with CK19 fragments in adenocarcinomas, with high significance and good correlation (rho $=0.5145, P=0.0036)$. However, further studies are required to understand this strong association on epithelial differentiation in metastatic pleural fluids from adenocarcinomas.
\end{abstract}

\section{Introduction}

Metastatic diseases are a predominant cause of pleural effusions in lung cancer. A malignant pleural effusion (MPE) may be the initial signal of lung cancer in $10-50 \%$ of patients with stage IV disease, according to the tumor, node, and metastasis (TNM) staging system [1]. Carcinomas of the lung and breast and lymphomas account for approximately $75 \%$ of MPE cases [1]. Lung epithelial cancer types, such as adenocarcinoma, squamous-cell carcinoma, and neuroendocrine tumors, are associated with the highest levels of biomarkers in serum and pleural fluid [2]. Cancer biomarkers are substances that are usually secreted by malignant cells or by the host in response to a tumor [3].

Adenosine deaminase (ADA) is a biomarker of pleural tuberculosis. However, the main function of this enzyme is to trigger the immune system in humans. Moreover, ADA also appears to be associated with neurotransmission, gestation, and the differentiation of epithelial cells [4]. Cytokeratins (CKs) are cancer biomarkers and are 
the main structural elements of the cytoskeleton in epithelial cells. CK19 is expressed in the epithelium covering the bronchial tree and is overexpressed in lung cancer [5]. Conflicting information on the relationship between $\mathrm{ADA}$ and cancer has been reported in previous studies, some of which have found ADA activity to be increased in malignant tissues and others of which have found it to be decreased [6-8].

ADA (E.C. 3.5.4.4) is an enzyme of the purine pathway that catalyzes the deamination of adenosine and $2^{\prime}$-deoxyadenosine into inosine and $2^{\prime}$-deoxyinosine, respectively. The conversion of inosine leads to hypoxanthine and uric acid or other mononucleosides. ADA is intracellularly expressed on the cell membrane. It does not have its own transmembrane domain and is therefore associated with a surface glycoprotein with two molecules of dipeptidyl peptidase IV, called CD 26. ADA is released by growth factors and cytokines, such as IL-2, IL-12, and interferon-gamma, and exhibits increased levels during malignancy [4].

CKs constitute an intermediate filament group that represents more than 20 different types of polypeptides, which are the major components of the cytoskeleton, a proteinaceous structural framework within the cellular cytoplasm. They are expressed in various sets, according to the epithelial type and the degree of differentiation. In normal human cells, CKs are classified as acidic type I (CK9-CK23) or neutralbasic type II (CK1-CK8). CK19 belongs to the class of type I cytokeratins. In cancer diagnosis, keratins are useful as diagnostic and prognostic biomarkers, active regulators of epithelial tumorigenesis, and indicators of treatment responsiveness [9-13]. Cytokeratin 19 fragments (CK19) are expressed in almost all epithelial malignancies, including breast cancer, lung cancer, colorectal carcinoma, cervical carcinoma, and papillary thyroid carcinoma. Its main functions are the maintenance of epithelial cell integrity, the mediation of stress responses, cell signaling, apoptosis, and participation in the immune response, including extravasation, migration, signaling, antigen recognition, phagocytosis, and cellular activation [9-13]. We hypothesized that a relationship could exist between pleural ADA and CK19 in patients with malignant pleural effusion, which has not been previously reported. Therefore, the objective of our study was to investigate the simultaneous occurrence of ADA and CK19 in malignant pleural fluids.

\section{Materials and Methods}

2.1. Design and Study Population. This was a prospective study conducted from January 2014 to January 2016 at Antonio Pedro Hospital, a teaching center of Fluminense Federal University, located in Niteroi, and General Hospital Santa Teresa, located in Petropolis, both in the State of Rio de Janeiro, Brazil. The Antonio Pedro Hospital Ethics Committee approved this study under the number $80 / 02$, according to the guidelines of the Helsinki Declaration. Written consent was obtained from all patients.

2.2. Inclusion and Exclusion Criteria for Patients in the Study. Pleural fluid samples were collected from continuous untreated individuals with pleural effusion syndrome due to several causes. The diagnosis of the cause of pleural effusion syndrome (PES) was confirmed by means of standard examinations and the use of appropriate surgical procedures [14]. The first biochemical tests used to diagnose a pleural transudate or exudate were current criteria for the dosage of total proteins and lactate dehydrogenase only in pleural fluids [15]. When a causal diagnosis of PES was unconfirmed after a thoracentesis procedure with laboratory evaluation of the pleural fluid, a closed-needle pleural biopsy was performed using Cope's needle. If PES persisted and symptoms increased or when it was not possible to differentiate malignancy and tuberculosis, the patient was referred for videoassisted thoracoscopic surgery [14]. The exclusion criteria included absolute contraindications, refusal to accept thoracentesis or other invasive procedures, use of immunosuppressive medications, hemolysis in pleural liquids, renal failure, HIV infection, and pleural effusion of an unknown cause. Patients with serum levels of bilirubin greater than $65 \mathrm{mg} / \mathrm{dL}$, lipid content great than $1500 \mathrm{mg} / \mathrm{dL}$, and a rheumatoid factor concentration great than $1500 \mathrm{IU} / \mathrm{M}$ were excluded from our study because these factors interfere with biomarker levels $[16,17]$.

2.3. ADA Assay. ADA assays, in which ADA irreversibly catalyzes the conversion of adenosine into inosine, with hydrogen peroxide produced in the final enzymatic reaction, were performed using a commercial kit. The assay is ready to use for automated chemistry analyzers using the kinetic method. Its principle relies on the detection of $\mathrm{H}_{2} \mathrm{O}_{2}$, and it is more sensitive than the colorimetric method of Giusti and Galanti. The assay is based on the Berthelot reaction, in which a blue dye produced by phenol-sodium hypochlorite is used to analyze the concentration of ammonia in pleural fluids. One unit of ADA is defined as the amount of ADA that generates one $\mu \mathrm{mol}$ of inosine from adenosine per min at $37^{\circ} \mathrm{C}[16]$.

2.4. Cytokeratin 19 Assay. The assay was performed using a CYFRA 21-1 kit designed to quantify soluble CK19 using an electrochemiluminescence immunoassay (ECLIA). The cut-off limit of the ECLIA in serum and plasma is $3.3 \mathrm{ng} / \mathrm{mL}$ with a specificity of $95 \%$. The assay has the ability to detect the tris $\left(2,2^{\prime}\right.$-bipyridyl $)$ ruthenium complex, an electrochemically luminescent molecule. The technique can be used for sandwich format and competitive immunoassays. CYFRA 21-1 is identified by two specific mouse monoclonal antibodies (Ks 19-1 and BM 19-21). These antibodies are directed toward two different antigenic determinants of a fragment of CK19. The sandwich complex binds to the particulate solid phase through the interaction of biotin and streptavidin. The reaction mixture is then aspirated into the measuring cell. The microparticles are magnetically captured on the electrode. The chemiluminescence emission is induced by applying voltage to the electrode and is measured by a photomultiplier [17]. The pleural fluids for the CYFRA 21-1 and ADA assays were stored at $-20^{\circ} \mathrm{C}$. CYFRA $21-1$ and $\mathrm{ADA}$ in the pleural fluid were determined in a blinded manner, without information on the definitive diagnosis. 
2.5. Statistical Approach. Both descriptive and inferential statistics were calculated using GraphPad (GraphPad Software Inc., version 6.0, La Jolla, CA, USA). The laboratory data were analyzed by means of a univariate analysis. A $P$ value less than 0.05 determined from a two-tailed test was considered statistically significant, to reject the null hypothesis with $5 \%$ probability of a type I error. The Shapiro-Wilk test was used to assess the normality of the data. The quantitative variables were assumed to be normally distributed and expressed in terms of their means and standard deviations (SDs), and those with nonnormal distributions were expressed in terms of their medians and interquartile ranges (IQRs). Qualitative or categorical variables were expressed in terms of proportions. To compare the information on MPE and benign pleural effusions (BPEs) between the two groups, data were analyzed using Student's $t$-test if the data were normally distributed or using the Mann-Whitney $U$ test if the data did not follow a normal distribution. The chi-squared test was used to compare proportions, as recommended by Campbell (2007) and Richardson (2011). The Kruskal-Wallis $H$ test (KW test) and post hoc Dunn test were used to compare ADA and CK19 levels in the adenocarcinoma group versus three or more unpaired (independent) samples in the several groups studied. The correlations between the ADA and CK19 results and several causes of pleural effusion were calculated using Spearman's rank correlation coefficient rho, which is commonly used for nonparametric correlation. By convention, correlation coefficients above 0.70 represent strong correlation, values between 0.50 and 0.70 represent good correlation, values between 0.30 and 0.50 represent moderate correlation, and values less than 0.30 represent weak correlation [18].

\section{Results}

The sample size used in this study was 119 pleural fluid samples from untreated individuals with pleural effusion syndrome due to several causes. The demographic characteristics of the patients are summarized in Table 1. In comparison to those in the BPE group, the CK19 fragment levels in the MPE group were significantly increased (median level: $85.03 \mathrm{ng} / \mathrm{mL}$ versus $13.41 \mathrm{ng} / \mathrm{mL}, P=0.0001$ ).

The patients were separated into eight subgroups of benign and malignant pleural fluids (Table 2): adenocarcinomas of different sites $(n=30)$, tuberculosis $(n=28)$, transudates by congestive heart failure $(n=21)$, simple and complicated parapneumonic effusion $(n=15)$, nontuberculous empyema $(n=8)$, squamous-cell carcinomas of different sites $(n=7)$, lymphomas $(n=4)$, and other causes $(n=6)$, including Dressler syndrome $(n=3)$, melanocarcinoma $(n=1)$, chylothorax $(n=1)$, and leukemia $(n=1)$. Differences in the medians of the ADA and CK19 levels in the several groups of pleural fluid were statistically significant (Kruskal-Wallis test, $P<0.0001$ ), as shown in Table 2 .

ADA levels exhibited a significant $P$ value (Dunn's test, $P<0.05)$ in patients with tuberculosis versus adenocarcinoma, as well as parapneumonic and transudative pleural fluid. However, the $P$ value according to Dunn's test was not significant $(P>0.05)$ for tuberculosis versus other pleural fluids: mainly empyemas, lymphomas, and squamous-cell carcinomas (Table 2).

The CK19 analysis helped us to exclude adenocarcinomas versus tuberculosis, transudates, and parapneumonic effusions with a highly significant $P$ value (Dunn's test, $P<0.0001$ ), as shown in Table 2. For the remaining pleural fluids, the $P$ value with Dunn's test was not significant $(P>0.05)$.

Finally, we determined the value of Spearman's correlation coefficient between adenosine deaminase and cytokeratin 19 fragments in the pleural fluids of the 119 patients with final diagnoses. As indicated in Table 3, ADA levels were significantly positively correlated with CK19 fragments in adenocarcinomas (rho $=0.5145, P=0.0036$, Figure 1 ) and significantly negatively correlated with other diseases (rho $=$ $-0.9429, P=0.0167)$. No significant correlations were found with tuberculosis (rho $=-0.9614, P=0.6265$ ), transudates (rho $=0.1805, P=0.4336$ ), parapneumonics $(\mathrm{rho}=-0.1321$, $P=0.6387)$ empyemas $\quad(r h o=-0.5663, \quad P=0.1511)$, squamous-cell carcinomas (rho $=0.0714, P=0.9063$ ), or lymphomas ( $\mathrm{rho}=0.4000, P=0.7500$ ).

\section{Discussion}

Malignant pleural fluids may be useful in modeling the hierarchical progression and heterogeneity of lung cancer [19]. The results of the present study provide the first evidence that increased levels of CK19 in pleural fluids are associated with adenosine deaminase activity in malignant pleural effusions from adenocarcinomas.

A paper recently published by our group described a study in which patients with benign pleural effusions were predominantly male [20]. The median and range of patients' ages in that study were similar to those shown in Table 1. Tuberculosis and adenocarcinomas are the most frequent causes of exudative pleural effusions in Brazil (Table 2). Table 2 shows the determination of cytokeratin 19 fragments and ADA activity in the pleural fluid of the patients in this study presenting with confirmed pleural effusion syndrome from any of several origins, mainly adenocarcinoma and other diseases such as tuberculosis. ADA is undoubtedly the best biomarker for pleural tuberculosis in clinical practice [20]. Our study, similar to many others, shows that the ADA level is useful in the differential diagnosis between tuberculosis and adenocarcinoma in pleural fluids. As shown in Table 2, the CYFRA 21-1 assay detected circulating fragments of cytokeratin 19 in adenocarcinomas and squamous-cell carcinomas, and the difference between pleural tuberculosis and other diseases was significant $(\mathrm{KW}=37.19, P<0.0001)$. In clinical practice, CK19 is a useful biomarker for the diagnosis of lung cancer [21]. However, it is important to remember that diagnostic decisions regarding MPE should depend on conclusive cytology results for pleural fluid or pleural biopsy [22].

We attempted to explore the possible causes for the high levels of CK19 in lung cancer. Human lung cancer cells in pleural fluids have been found to be more invasive than cancer cells from primary sites. This difference may be related to epithelial-mesenchymal transition (EMT). EMT 
TABLE 1: Demographic characteristics, ADA levels, and CK19 of controls (benign pleural effusions) and malignant pleural effusions (cases) in 119 patients.

\begin{tabular}{lccc}
\hline Demographic characteristics and pleural biomarkers & MPE $(n=43)$ & BPE $(n=76)$ & Test $(P$ value $)$ \\
\hline Male, $n(\%)$ & $15(35.0)$ & $36(47.0)$ & Chi-squared $=0.608(P=0.4354)$ \\
Female, $n(\%)$ & $28(65.0)$ & $40(53.0)$ & Chi-squared $=0.959(P=0.3275)$ \\
Mean age (years \pm SD) & $62.33 \pm 12.37$ & $60.95 \pm 21.37$ & $T=1.323(P=0.1931)$ \\
Median total ADA levels (IU/L) in normal scale (IQR) & $9.76(6.74-13.90)$ & $11.63(6.64-34.32)$ & $U=1407.0(P=0.2102)$ \\
Median CK19 levels (ng/mL) in normal scale (IQR) & $85.03(24.61-362.3)$ & $13.41(7.06-32.30)$ & $U=586.0(P=0.0001)$ \\
\hline
\end{tabular}

Abbreviations: MPE: malignant pleural effusion; BPE: benign pleural effusion; SD: standard deviation; ADA: adenosine deaminase; CK19: cytokeratin 19 fragments; IQR: interquartile range, 25th-75th percentiles.

TABle 2: Adenosine deaminase and cytokeratin 19 fragment levels in the pleural fluids of 119 patients with final diagnoses.

\begin{tabular}{lccc}
\hline Diseases & Patients $(n)$ & Adenosine deaminase, median (IU/L) (IQR) & Cytokeratin 19 fragments, median $(\mathrm{ng} / \mathrm{mL})(\mathrm{IQR})$ \\
\hline Adenocarcinomas & 30 & $9.41(6.56-13.31)$ & $98.96(23.76-476.6)$ \\
Tuberculosis & 28 & $39.08(26.66-45.96)$ & $16.72(8.58-34.72)$ \\
Transudates & 21 & $3.26(2.09-7.73)$ & $8.40(5.91-13.94)$ \\
Parapneumonics & 15 & $9.38(5.68-9.97)$ & $12.09(6.06-28.86)$ \\
Empyemas & 08 & $32.94(16.07-61.70)$ & $24.55(2.03-205.3)$ \\
Squamous cell carcinomas & 07 & $13.11(11.08-18.65)$ & $52.11(24.63-236.2)$ \\
Lymphomas & 04 & $21.64(10.0-750.9)$ & $14.99(3.96-60.86)$ \\
Other & 06 & $10.14(5.26-24.30)$ & $27.43(14.61-32.40)$ \\
\hline
\end{tabular}

Abbreviations: IQR: interquartile range; ADA: adenosine deaminase; CK19: cytokeratin 19 fragments. Other diseases: Dressler syndrome $(n=3)$, melanocarcinoma $(n=1)$, chylothorax $(n=1)$, and leukemia $(n=1)$. Kruskal-Wallis test: $H=63.10$ for ADA $(P<0.0001)$ with $P<0.05$ for tuberculosis versus adenocarcinoma, parapneumonic, and transudate; $P>0.05$ for tuberculosis versus other groups. For CK19, $H=37.19(P<0.0001)$ with $P<0.05$ for adenocarcinoma versus tuberculosis, transudate, and parapneumonic. Diseases remaining: $P>0.05$.

TABLE 3: Spearman's rank correlation coefficients (rho) between adenosine deaminase and cytokeratin 19 fragments in the pleural fluids of 119 patients with final diagnoses.

\begin{tabular}{lccc}
\hline Diseases & Rho & $\begin{array}{c}\text { 95\% confidence } \\
\text { intervals }\end{array}$ & $\begin{array}{c}P \text { value } \\
\text { (two tailed) }\end{array}$ \\
\hline Adenocarcinomas & 0.5145 & $0.178-0.743$ & 0.0036 \\
Tuberculosis & -0.9614 & -0.466 to 0.297 & 0.6265 \\
Transudates & 0.1805 & -0.285 to 0.577 & 0.4336 \\
Parapneumonics & -0.1321 & -0.614 to 0.421 & 0.6387 \\
Empyemas & -0.5663 & -0.908 to 0.230 & 0.1511 \\
Squamous cell & 0.07143 & -0.720 to 0.782 & 0.9063 \\
carcinomas & & & \\
Lymphomas & 0.4000 & -0.911 to 0.983 & 0.7500 \\
Other & -0.9429 & -0.993 to 0.560 & 0.0167 \\
\hline
\end{tabular}

Interpretation of rho: values above 0.70 , strong correlation; $0.50-0.70$, good correlation; 0.3-0.5, moderate correlation; and less than 0.30, poor correlation.

is reactivated during adulthood under conditions of disease, such as cancer. EMT determines the severity of cancer and contributes to metastasis [5]. EMT is a progressive malignant cell program that enhances the mobility, invasion, resistance to apoptotic stimuli, and therapeutic resistance of cancer cells [23]. Levels of CK19 may reflect cytoskeleton formation in malignant cells and the association with the degree of cancer differentiation toward the squamous epithelium. The keratin content increases when epithelial cells transform into cancer cells. Soluble fragments of CK19 are released into the pleural fluid as a result of necrosis or apoptosis of malignant cells with increased caspase-3 activity [24].

Spearman's correlation coefficients obtained for the correlations between adenosine deaminase and cytokeratin 19 fragments in pleural fluids were significantly positive for adenocarcinomas and significantly negative for a heterogeneous group of other diseases, including Dressler syndrome, melanocarcinoma, chylothorax, and leukemia, as shown in Table 3. These findings indicate a highly significant relationship between these two biomarkers, mainly in the pleural fluids from adenocarcinomas (Figure 1). There was a good correlation or relationship between ADA and CK19 in pleural adenocarcinomas $(n=30)$ : rho $=0.5145$ and $P=0.0036$ (Table 3 ). There is a tendency to believe that a correlation between biomarkers means that one causes or influences changes in the other. However, a correlation does not imply causation, and an unknown third factor can be responsible for fluctuations in both ADA and CK19 in pleural adenocarcinoma cells. Furthermore, what is the explanation for the significant correlation between the levels of ADA and CK19 fragments in pleural adenocarcinoma? We could speculate that cancer cells contain high levels of extracellular adenosine. When binding to its receptors, the extracellular adenosine exhibits angiogenic and anti-inflammatory actions. It also exhibits intracellular action that modulates purine levels and affects tumor growth and metastatic phenotype [25]. Therefore, a need for increased adenosine in adenocarcinoma cells leads to increased ADA production (Table 3). 


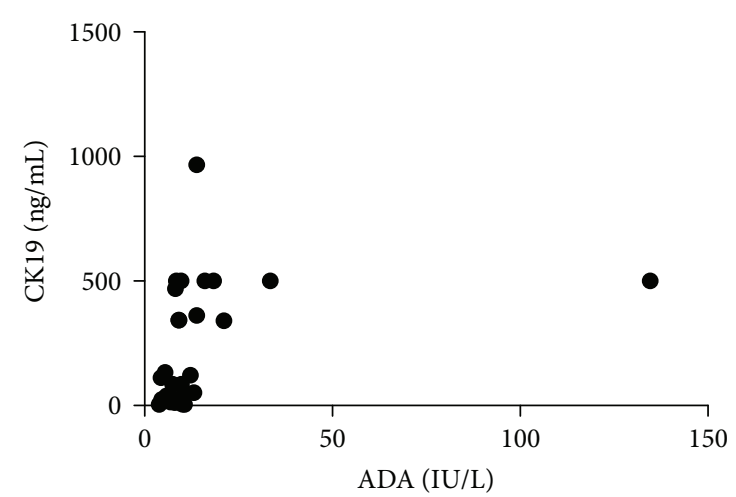

FIGURE 1: Spearman's correlation between CK19 fragments (ng/mL) and $\mathrm{ADA}$ levels (IU/L) in adenocarcinoma groups $(n=30$, rho $=0.5145, P=0.0036$ ).

Limitations of the study. The interpretation of this study have been considered in relation to the controversial results in the literature to elucidate the usefulness of tumor biomarkers in malignant pleural effusions when the site of primary cancer is unknown [26, 27]. However, the use of a biomarker for diagnosis always limits its result interpretation on a previous analysis of clinical manifestations, image findings, epidemiological profile of the evaluated patient, and a false-positive level for other possible diseases [20].

\section{Conclusions}

The results of our study demonstrate a strong association between ADA and CK19 levels in pleural metastatic adenocarcinomas. However, further studies are required to fully understand whether adenosine deaminase and cytokeratin 19 fragments play the same important role in epithelial differentiation in metastatic pleural fluids from adenocarcinomas.

\section{Disclosure}

The authors have no financial involvement with any organization or entity with a financial interest in the subject matter or materials discussed in the manuscript. This includes employment, consultancies, honoraria, stock ownership or options, expert testimony, grants or patents received or pending, or royalties.

\section{Conflicts of Interest}

The authors declare that they have no conflicts of interest.

\section{References}

[1] J. M. Porcel, M. M. Lui, A. D. Lerner, H. E. Davies, D. FellerKopman, and Y. C. G. Lee, "Comparing approaches to the management of malignant pleural effusions," Expert Review of Respiratory Medicine, vol. 11, no. 4, pp. 273-284, 2017.

[2] W. D. Travis, E. Brambilla, A. G. Nicholson et al., "The 2015 World Health Organization classification of lung tumors: impact of genetic, clinical and radiologic advances since the
2004 classification," Journal of Thoracic Oncology, vol. 10, no. 9, pp. 1243-1260, 2015.

[3] M. Gion, C. Trevisiol, A. W. S. Rutjes, G. Rainato, and A. S. C. Fabricio, "Circulating tumor markers: a guide to their appropriate clinical use comparative summary of recommendations from clinical practice guidelines (PART 3)," The International Journal of Biological Markers, vol. 32, no. 2, pp. e147-ee81, 2017.

[4] Y. Moriwaki, T. Yamamoto, and K. Higashino, "Enzymes involved in purine metabolism-a review of histochemical localization and functional implications," Histology and Histopathology., vol. 14, no. 4, pp. 1321-1340, 1999.

[5] D. Huang, W. Xu, X. Xu, X. Zhang, R. Zhou, and P. Chen, "EMT influences the expression of CK19 in pleural effusionderived lung cancer cells and their invasion and metastasis," Oncology Letters, vol. 12, no. 6, pp. 5052-5058, 2016.

[6] D. C. Kelgandre, J. Pathak, S. Patel, P. Ingale, and N. Swain, "Adenosine deaminase - a novel diagnostic and prognostic biomarker for oral squamous cell carcinoma," Asian Pacific Journal of Cancer Prevention, vol. 17, no. 4, pp. 1865-1868, 2016.

[7] M. E. Balis, "Adenosine deaminase and malignant cells," Annals of the New York Academy of Sciences, vol. 451, no. 1, pp. 142-149, 1985.

[8] N. Pirinççi, T. Y. Kaya, M. Kaba et al., "Serum adenosine deaminase, catalase, and carbonic anhydrase activities in patients with renal cell carcinoma," Redox Report, vol. 22, no. 6, pp. 252-256, 2017.

[9] J. K. Burkhardt, "Cytoskeletal function in the immune system," Immunological Reviews, vol. 256, no. 1, pp. 5-9, 2013.

[10] M. Miédougé, A. Devys, M. Simon et al., "High levels of cytokeratin 19 fragments but no evidence of cytokeratins $1,2,10$ / 11,14 or filaggrin in the serum of squamous cell lung carcinoma patients," Tumour Biology, vol. 22, no. 1, pp. 19-26, 2001.

[11] R. Moll, M. Divo, and L. Langbein, "The human keratins: biology and pathology," Histochemistry and Cell Biology, vol. 129, no. 6, pp. 705-733, 2008.

[12] V. Karantza, "Keratins in health and cancer: more than mere epithelial cell markers," Oncogene, vol. 30, no. 2, pp. 127138, 2011.

[13] A. T. Ritter, K. L. Angus, and G. M. Griffiths, "The role of the cytoskeleton at the immunological synapse," Immunological Reviews, vol. 256, no. 1, pp. 107-117, 2013.

[14] C. Hooper, Y. C. G. Lee, N. Maskell, and on behalf of the BTS Pleural Guideline Group, "Investigation of a unilateral pleural effusion in adults: British Thoracic Society pleural disease guideline 2010," Thorax, vol. 65, Supplement 2, pp. ii4-i17, 2010.

[15] B. H. Maranhao, C. T. Silva Junior, A. M. Chibante, and G. P. Cardoso, "Determination of total proteins and lactate dehydrogenase for the diagnosis of pleural transudates and exudates: redefining the classical criterion with a new statistical approach," Jornal Brasileiro de Pneumologia, vol. 36, no. 4, pp. 468-474, 2010.

[16] H. Delacour, C. Sauvanet, F. Ceppa, and P. Burnat, “Analytical performances of the diazyme ADA assay on the Cobas ${ }^{\circledR} 6000$ system," Clinical Biochemistry, vol. 43, no. 18, pp. 14681471, 2010.

[17] H. Bodenmuller, B. Ofenloch-Hahnle, E. B. Lane, A. Dessauer, V. Bottger, and F. Donie, "Lung cancer-associated keratin 19 
fragments: development and biochemical characterisation of the new serum assay Enzymun-Test ${ }^{\circledR}$ Cyfra 21-1," The International Journal of Biological Markers, vol. 9, no. 2, pp. 75-81, 1994.

[18] A. Hazra and N. Gogtay, "Biostatistics series module 6: correlation and linear regression," Indian journal of dermatology., vol. 61, no. 6, pp. 593-601, 2016.

[19] S. K. Basak, M. S. Veena, S. Oh et al., "The malignant pleural effusion as a model to investigate intratumoral heterogeneity in lung cancer," PLoS One, vol. 4, no. 6, article e5884, 2009.

[20] R. F. Behrsin, C. T. Junior, G. P. Cardoso, J. L. Barillo, J. B. de Souza, and E. G. de Araujo, "Combined evaluation of adenosine deaminase level and histopathological findings from pleural biopsy with Cope's needle for the diagnosis of tuberculous pleurisy," International Journal of Clinical \& Experimental Pathology, vol. 8, no. 6, pp. 7239-7246, 2015.

[21] W. W. Huang, S. M. Tsao, C. L. Lai, C. C. Su, and C. E. Tseng, "Diagnostic value of Her-2/neu, Cyfra 21-1, and carcinoembryonic antigen levels in malignant pleural effusions of lung adenocarcinoma," Pathology, vol. 42, no. 3, pp. 224-228, 2010.

[22] Y. Yang, Y. L. Liu, and H. Z. Shi, "Diagnostic accuracy of combinations of tumor markers for malignant pleural effusion: an updated meta-analysis," Respiration, vol. 94, no. 1, pp. 62-69, 2017.

[23] V. Mittal, "Epithelial mesenchymal transition in aggressive lung cancers," Advances in Experimental Medicine and Biology, vol. 890, pp. 37-56, 2016.

[24] S. Linder, "Cytokeratin markers come of age," Tumour Biology, vol. 28, no. 4, pp. 189-195, 2007.

[25] S. S. Virtanen, A. Kukkonen-Macchi, M. Vainio et al., "Adenosine inhibits tumor cell invasion via receptor-independent mechanisms," Molecular Cancer Research, vol. 12, no. 12, pp. 1863-1874, 2014.

[26] H. Ustün, A. Borazan, N. Bilgiçli, and A. Yilmaz, "Diagnostic value of tumoural markers in pleural effusions," International Journal of Clinical Practice, vol. 58, no. 1, pp. 22-25, 2004.

[27] L. Antonangelo, R. K. Sales, A. P. Corá, M. M. Acencio, L. R. Teixeira, and F. S. Vargas, "Pleural fluid tumour markers in malignant pleural effusion with inconclusive cytologic results," Current Oncology, vol. 22, no. 5, pp. e336-e341, 2015. 


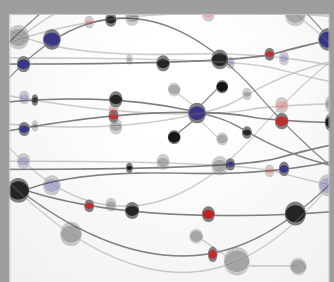

The Scientific World Journal
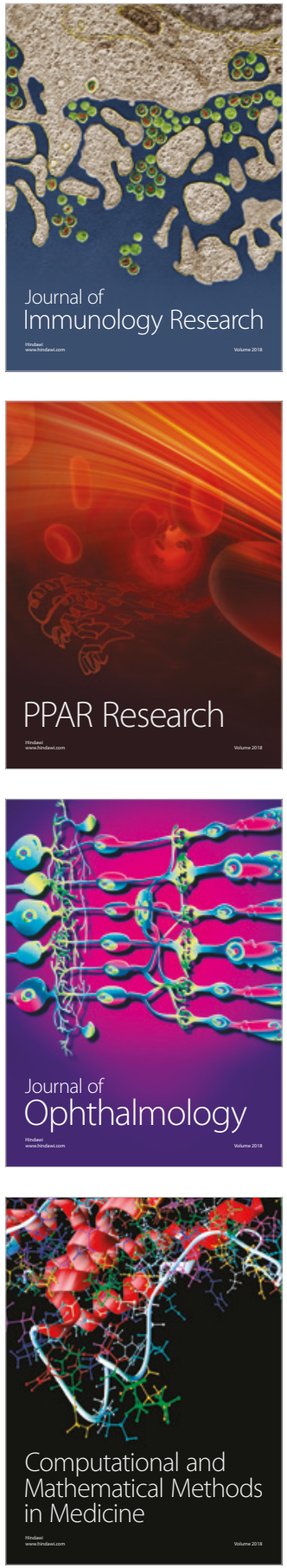

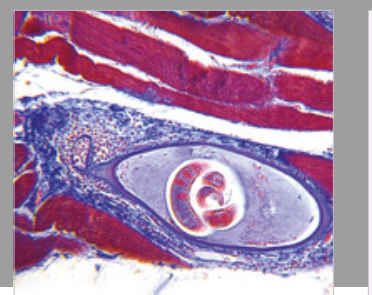

Gastroenterology Research and Practice

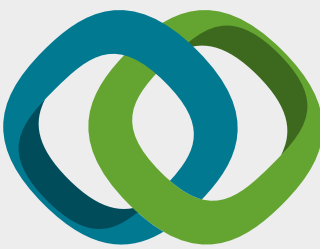

\section{Hindawi}

Submit your manuscripts at

www.hindawi.com
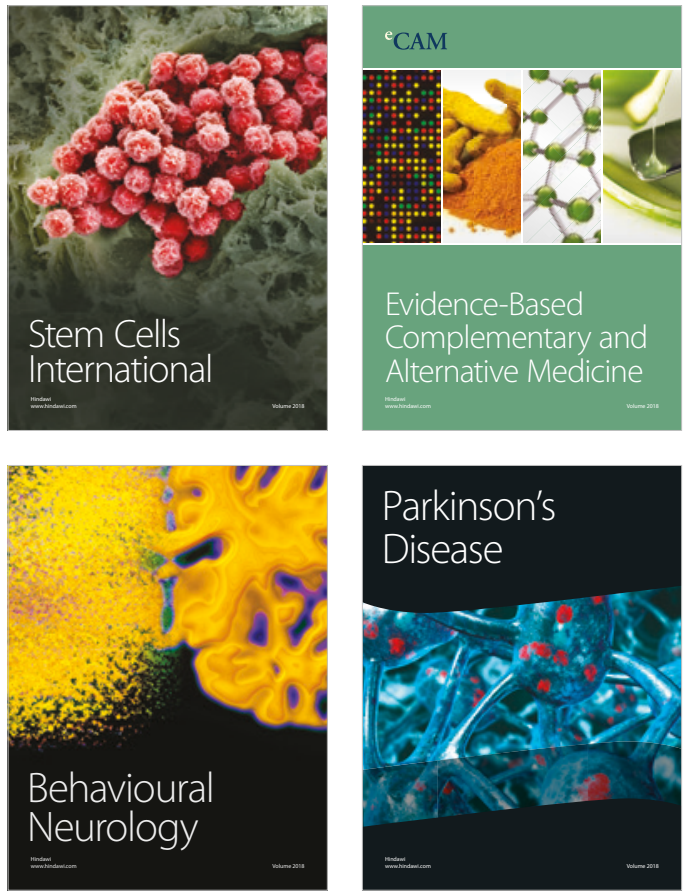

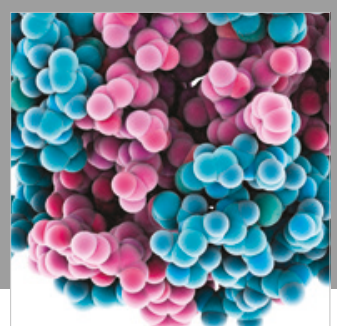

ournal of

Diabetes Research

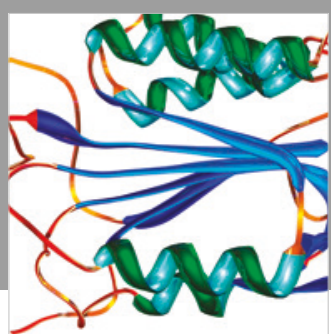

Disease Markers
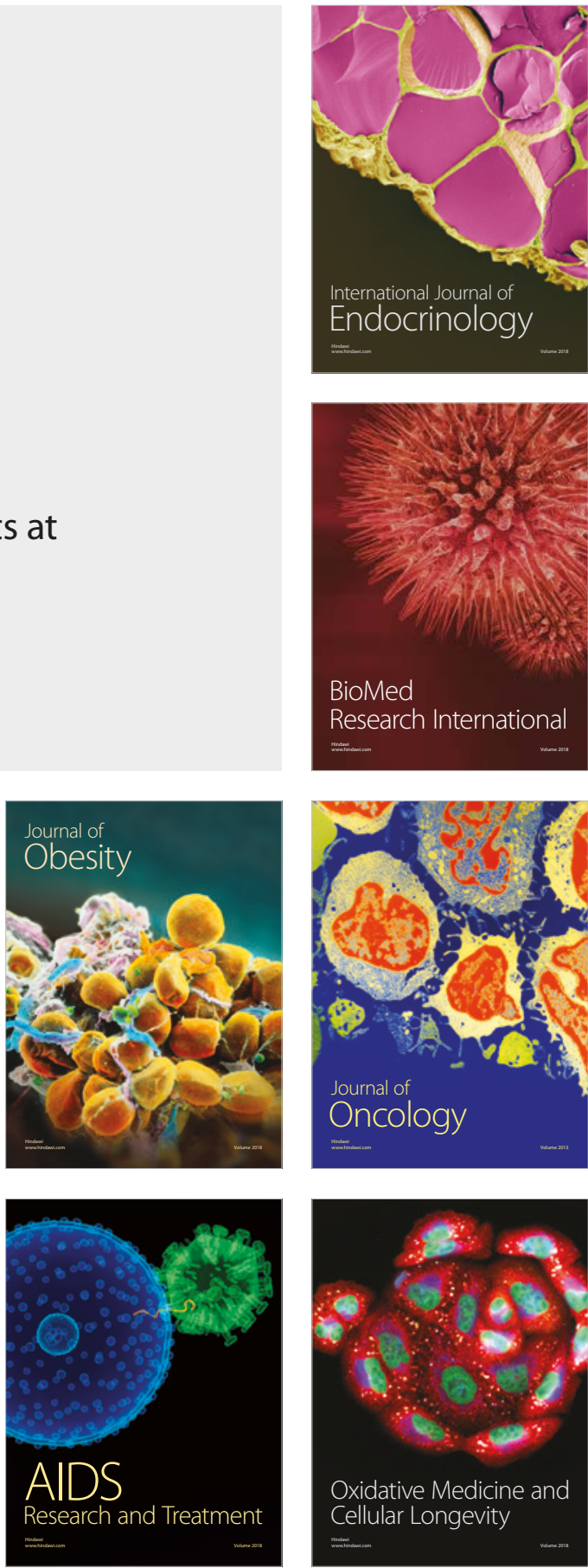